\title{
Retraction Note: A mathematical model to study the relationship between the dietary profiles, core body temperature and sleep profile in the high school students
}

\author{
Siddhanta Panda ${ }^{1} \cdot$ Trina Sengupta $^{2} \cdot$ Tandeep Kaur $^{1}$
}

Published online: 24 October 2019

(c) Japanese Society of Sleep Research 2019

\section{Retraction Note: Sleep and Biological Rhythms (2018) 16: 307-313 https://doi. org/10.1007/s41105-018-0157-7}

The editors have retracted this article [1] because they remain unconvinced by the provenance, ownership and reliability of the data that the authors claim to have collected using the application 'myCircadianClock' within this study. As a consequence, the conclusions within this publication are unreliable. In addition, it did not have ethical oversight from a relevant ethics committee. None of the authors agree to this retraction.

\section{Reference}

1. Panda S, Sengupta T, Kaur T. A mathematical model to study the relationship between the dietary profiles, core body temperature and sleep profile in the high school students. Sleep Biol Rhythms. 2018;16:307-13.

The original article can be found online at https://doi.org/10.1007/ s41105-018-0157-7.

Trina Sengupta

sengupta.trina3@gmail.com

Siddhanta Panda

panda.siddhanta@yahoo.in

Tandeep Kaur

tandeepkaur@yahoo.com

Delhi Public School (DPS), RK Puram, New Delhi, India

2 Baldev Singh Sleep Laboratory, Department of Physiology, All India Institute of Medical Sciences (AIIMS), New Delhi, India 